\title{
Presentación
}

$\mathrm{E}$ 1 Dr. Irving Selikoff (1915-1992) fue uno de los más connotados líderes mundiales en el campo de la salud ambiental y ocupacional. Su vida estuvo ligada a la Escuela de Medicina de Monte Sinaí, donde ocupó diversos cargos y al final de su carrera fue designado Profesor Emérito. Durante su larga trayectoria académica recibió muchas distinciones, incluyendo el Premio Lasker en medicina. Su obra publicada es de casi 400 trabajos, lo que incluye artículos, monografías, capítulos y libros. Fue el fundador de la revista científica Environmental Research y editor de varias otras.

Entre los trabajos del Dr. Selikoff destacan los relacionados con los efectos adversos a la salud derivados de la exposición a los polvos de asbesto. Según el Dr. Herbert K. Abrams, la historia del asbesto ha tenido muchos protagonistas; desde el principo de este siglo se estudiaron las relaciones entre el asbesto y la tuberculosis. Poco a poco se fue evidenciando la necesidad de proteger a los trabajadores expuestos al asbesto y a las tierras diatomáceas, pero tocó al Dr. Selikoff despertar el amplio interés público y tomar parte en los juicios que definieron el marco legal para el control de las enfermedades relacionadas ocupacionalmente con el asbesto.

El artículo titulado Exposición al asbesto, tabaquismo y neoplasia, que publicara el Dr. Selikoff en la prestigiada revista Journal of the American Medical Association en 1968, es sin duda un trabajo clásico de la investigación en salud. Su experiencia clínica y de investigador, trabajando junto con los sindicatos, le había dado ya un profundo conocimiento acerca de los tipos de daño que el asbesto ocasiona al ser humano; en particular, se había interesado en el hecho de que el asbesto parecía inducir varios tipos de tumores cancerosos, pero especialmente cánceres de las vías respiratorias bajas.

Hasta antes de publicar el artículo que nos ocupa, quedaba la duda de que el exceso de cánceres pulmonares fuese debido al consumo de tabaco y no tanto a un efecto independiente del asbesto. Hoy en día entendemos perfectamente esta preocupación, que corresponde a la necesidad de tomar en cuenta a las terceras variables como explicación alternativa de los efectos observados, es decir la presencia de confusión en los datos de un estudio epidemiológico. Pero el Dr. Selikoff fue mucho más allá; se adelantó a su tiempo al imaginar que estuviese presentándose un fenómeno de alta complejidad que ahora llamamos modificación del efecto en la epidemiología moderna. El razonamiento, a final de cuentas correcto, del Dr. Selikoff se basó en la aceptación de algo todavía fuertemente cuestionado en aquéllos tiempos, que era la asociación entre tabaquismo y cáncer pulmonar; según podemos leer en su texto, el Dr. Selikoff y sus colegas estuvieron más interesados en probar la hipótesis de que ambos factores tenían, al menos, efectos independientes, que en la posibilidad antes mencionada de que el tabaco fuese simplemente un confusor (aunque el concepto todavía no estuviese en boga).

Tal parece que el Dr. Selikoff no fue sorprendido por el hecho de que la exposición al tabaco y al asbesto produjese un efecto mayor que la simple conjunción de sus efectos independientes; si acaso lo sorprendió que el resultado alcanzara un valor tan alto. Pocos investigadores, aún ahora, se imaginan o se interesan en el asunto de la modificación del efecto. Los métodos modernos, a pesar de las grandes facilidades que confieren los programas de cómputo, siguen siendo tan complicados que la mayoría los pasa por alto. Es verdaderamente apasionante encontrar, paso a paso, en el texto la secuencia de las actividades y los hallazgos que permitieron a los autores esclarecer y adelantar las fronteras del conocimiento. 
Pocos de nosotros, si acaso alguno, podremos encontrar y explorar un tema tan importante, de una manera tan impecable. Honor a quien honor merece, el Dr. Irving Selikoff contribuyó grandemente a nuestro conocimiento y propició acciones gran trascendencia para proteger la salud humana.

Malaquías López Cervantes*

\section{Bibliografía consultada}

Abrams HK. Some hidden history of occupational medicine. Environ Res 1992;59:23-35.

Frank AL. A special issue dedicated to the memory of I. J. Selikoff. Environ Res 1992;59:1-22.

Maltoni C. The long-lasting legacy of industrial carcinogens: the lesson of asbestos. Irving J. Selikoff Memorial Lecture, 1995. Ann N Y Acad Sci 1997;837:570-86.

Markowitz S, et al. Innovation in international training in occupational and environmental health: challenging unspoken assumptions. Int J Occup Environ Health 1999;5:237-40.

Nicholson WJ, et al. Remembering Irving J. Selikoff. Mt Sinai J Med 1994;61:500-3.

Samuels SW. Philosophic perspectives: community, communications, and occupational disease causation. Int J Health Serv 1998;28:153-64.

Nicholson WJ, et al. Remembering Irving J. Selikoff. Mt Sinai J Med 1994;61:500-3.

Selikoff IJ, et al. The third epidemiological revolution. Eur J Epidemiol 1992;8:625-6.

Selikoff IJ. Asbestos disease-1990-2020: the risks of asbestos risk assessment. Toxicol Ind Health 1991;7:117-27.

Selikoff IJ, et al. A landmark case in asbestosis. JAMA 1991;265:898-901.

Selikoff IJ. Historical developments and perspectives in inorganic fiber toxicity in man. Environ Health Perspect 1990;88:269-76.

Selikoff IJ. Occupational disease: nature and extent. Keynote address. Ann N Y Acad Sci 1989; 572:4-9; discussion $55-60$.

* Centro de Investigación en Sistemas de Salud, Instituto Nacional de Salud Pública, México. 